\title{
Machine Monitoring Protocols Based on Quality of Service (QoS) to Improve Performance of Real-Time Industrial Applications
}

\author{
Emad H. Abualsauod (iD \\ Department of Industrial Engineering, College of Engineering, Taibah University, 41411 Madina Almonwara, Saudi Arabia \\ Correspondence should be addressed to Emad H. Abualsauod; eabualsauod@taibahu.edu.sa
}

Received 9 December 2021; Revised 23 December 2021; Accepted 27 December 2021; Published 31 January 2022

Academic Editor: Vijay Kumar

Copyright (C) 2022 Emad H. Abualsauod. This is an open access article distributed under the Creative Commons Attribution License, which permits unrestricted use, distribution, and reproduction in any medium, provided the original work is properly cited.

\begin{abstract}
A major factor in the optimization of real-time industrial applications is Quality of Service (QoS). There is also a quality-of-service parameter (QoS). Normally, dynamic networks require QoS settings for real-time applications. The industrial production environment is changing due to rapid advances in ICT. Industries have increased output due to improved manufacturing chain efficiency, flexibility, and security. Establishing a secure, reliable, and fast network in the sector is therefore critical. In industrial applications, data collection is an important factor to consider. Various routing methods exist for collecting data from industrial environments and sending it to processing. In this situation, the energy consumption of device nodes and data transmission delay are important factors to consider. This research proposes to construct an energy-efficient delay-aware WSN routing (EDR) protocol for defect detection. The clustering group optimal approach is used to identify which clusters should be selected. A modified task selection method is proposed to calculate the most efficient path between industrial areas and data centres (monitoring rooms). The node selection approach is used to find the network's aggregate node. The Network Simulator (NS2) programmed is used to model the projected IEM-EDR system. The proposed Industrial Energy Monitoring-Energy-Efficient Delay-Aware Routing (IEM-EDR) system's performance is compared to that of existing techniques in hop count, latency, energy consumption, throughput, and delivery rate.
\end{abstract}

\section{Introduction}

WSNs [1] were first utilized in military purposes, but when more sophisticated technology became available, they began to be used in industrial applications, such as assembly line monitoring and production automation. When used in industrial applications such as assembly line monitoring and factory automation, Wireless Sensor Networks (WSNs) may help to improve aspects such as efficiency, dependability, and productivity $[2,3]$. Industrial WSNs (IWSNs) are a form of WSNs that are used in the manufacturing industry [4]. Sensors that are geographically dispersed must adhere to stringent security and reliability requirements in real-time communications. The failure to preserve the security and reliability of sensed data transfers may have an impact on the production line, causing damage to the industrial equipment and possibly the loss of employees' lives if not addressed immediately [5].

Furthermore, in industrial contexts, the effects of metallic frictions, mechanical barriers, equipment noise, and engine vibrations are the polar opposite of those experienced during radio transmission in space. The performance of wireless communications will be hampered as a result of this [6]. In addition, since radio propagation is broadcast in nature, the wireless media is completely free to be used by both illegal and authorized users. As a result, input nodes are more susceptible to eavesdropping outbreaks than cable sensor networks. In this situation, the communication nodes are physically linked by wire cables, and a node that is not physically attached is impossible to contact a node that is connected for illicit operations [7]. As long as an eavesdropper remains hidden inside industrial WSNs, lawful 
wireless communications between sensors may be easily overheard by the eavesdropper, who may then decode taped messages, therefore violating the privacy of the sensors' information.

IWSN has fully fledged in relevance and is now employed in a wide range of applications [8, 9]. Critical infrastructure is a common application for IWSN, and it is used to create supervisory control and data acquisition (SCADA) systems [10]. Their acceptance in these systems is due to the fact that they provide the same control characteristics as their wired equivalents while incurring much lower maintenance and implementation costs. As part of the Internet of Things application ecosystem [11], IWSN is also utilized for intelligent monitoring and control. Because the Internet of Things (IoT) technologies are developing at a rapid pace, these devices have the potential to become key parts of smart city applications. According to the results of the poll, there might be more than 100 billion Internet of Things apps in use by 2020, with the market value of these projects now exceeding 11 billion Euro in Europe alone at the time of writing [12].

Given the complexity of the application environment, a diverse team of professionals is required to work on both the application and the development side of things together. In IWSNs, a circulated irregularity discovery outline [13] is occupied because soft segmentation of the information space is carried out by the use of the fuzzy $c$-means method in an incremental model, which is a soft partitioning of the data space. Similarly, to the FoF paradigm, sensor web enablement (SWE) [14] is another technology that is being utilized in indoor applications to monitor worker behavior as well as to ensure their health and safety. Volatile organic compounds (VOCs) [15] are being investigated for their possible use in industrial installations using an array of interdigitated chemocapacitors (IDCs) covered with semiselective polymers as sensing layers, which are being investigated. The FASyS system attempts to decrease potential labour hazards by identifying environmental elements that may be associated with such risks or accidents as early as feasible [16]. An indoor localization and position tracking system for pedestrians that implemented on a wearable embedded platform has been developed [17]. Three selective cooperative relaying protocols [18] were developed in conjunction with the aforementioned relay update techniques. This paper describes a method for mesh generation called AFECETS [19], which creates a mesh by using the Advancing Front Mesh Generation (AFMG) algorithm in conjunction with limited Delaunay triangulation and smoothing, as well as heuristics, to achieve an ideal topology. WSN tracking in an industrial context is accomplished via the use of a distributed, multiagent systems method that incorporates a model for battery depletion and replacement [20]. Z-curve surpasses both the SCAN and the HILBERT curves in terms of charging delay and travelling efficiency, and the S-curves and Z-curve [21] and examine the effects of pathways on energy provisioning in IWSNs with the alterations. The use of a multipath routing protocol [22] to ensure the rigorous Quality-of-Service requirements required. MPAR makes use of input estimations of the dependability and latency of various paths in order to determine the protuberances and ways that are required in order to construct end-to-end connections that provide the required levels of quality of service [23].

The majority of currently available location-aware recommendation algorithms are incompetent and tangled in a web of imbalanced alpha values. When developing recommendation systems, it has been discovered that a singledimensional approach, either by user dimension or by service dimension, has lower accuracy. Because of the absence of contextual information about the place, it is possible that unsuitable service suggestions may be made [24]. It is difficult to set a stable number for alpha, which represents the degree of relevance of an axis from an academic standpoint. Because there is no current validation mechanism that separates the request validation from the service rendering, the queue is overburdened with invalidated requests and unsecured hits, causing the queue to become congested. The smallest amount of work put into validating the present system leaves the service provider feeling uneasy and overburdened. Increasing the number of people in a highly ranked service provider's queue will lower the quality of service provider that is intended to be provided, and vice versa. According to the results of the survey, there is a need for a model that treats queue length as a random variable and that most likely picks a line that is shorter in length and provides the best effort service [25].

Six parts make up the body of the paper. The second part discusses the relevant work that has been done in connection with the data collection in the WSN. Section three of the report outlines the most serious issues with the current system. The suggested technique is detailed in detail in the fourth part of this document. Portion five contains the section containing the results. The last portion of the document contains the conclusion of the paper.

\section{Related Works}

Sheng et al. [26] have provided an impression of the Internet of Things ecosystem, as well as fundamental technologies that facilitate IoT communications, as well as the necessary management methods for an Internet of Things system. A cross-layer architecture with the well-organized and dependable administration of networks has been developed. The simplicity and efficiency of the solution are the performance assessments, and the solution is expected to be the driving force behind the standardization of IoT device management. These advantages allow the Internet of Things to overcome network complexities effectively and efficiently, while satisfying the needs of customers who want highquality products and services [27].

Researchers from across the globe are attempting to identify next-generation solutions for the Internet of Things, and Sun et al. [28] have suggested LR-WPAN infrastructures to meet the growing need for input and moveable connection in the IoT. Power management, energy-efficient design and operation, and low-power hardware and software are required for real-world implementations in complicated use cases. It is possible to adapt the engineering concepts and 
user interface synthesis outlined in this article for a broad variety of claims and use belongings, where major considerations are operating situations and input connectivity, quality of service, flexibility, charge, form factor, and energy competence [29]. Track and trace applications, position estimation applications, anomaly detection apps, theft detection applications, and sensor monitoring applications are some examples of what is available.

Sun et al. [28] developed the End-to-End Data Delivery Reliability Model (E2E-DDR model), which they describe as a truthful perfect of a WSN organized in a particular manufacturing context that is founded on realworld data. Aside from distance between transmitter and receiver nodes, an attempt is made to differentiate. In the E2E-DDR model, the distance between transmitter and receiver nodes is also taken into consideration. They have a considerable impact on differences in the wireless propagation behavior of all WSN nodes as well as on the dependability of data transmission in general [30]. In order to fulfil the rigorous reliability requirements of industrial monitoring and control systems, we may use the proposed model to predict the dependability of radio connections and, as a result, appropriately optimize a WSN during the design stage.

For manufacturing wireless sensor networks that contain mobile devices, Montero et al. [31] have designed and tested neighbor finding algorithms. The protocol takes use of info about the network architecture and the characteristics of devices in order to increase the ability to identify neighbor plans despite also using radio resources as effectively as possible. Because of the widespread industrial usage of the Wireless HART standard, protocols have been designed specifically for it. In any case, the suggested techniques may be modified input standards as they both keep wireless HART's passive listening approach for neighbor finding while also introducing an advertising scanning scheme with active solicitations for the ads. When compared to the wireless HART NDP solution, the study revealed that the suggested NDP protocols considerably enhance the ability of mobile devices to locate neighbor devices as well as the time required to detect them [32].

The fusion approaches were used by Kannan et al. [33] to suggest a reliable and energy-efficient framework for reliable data transfer based on the reliability of the transmission. The request for resources is made as a result of doing a thorough analysis of the data's qualities and attributes. When the suggested Multispectral Data Fusion Technique is used, it is possible to accomplish automated modification of the proportion of delivered messages, which is regarded an alternate method of increasing throughput while simultaneously decreasing energy usage. Selecting the core node from the set of sensor nodes, building an HPIS on the basis of time slices, and calculating boundary values all contributed to determining the core node's position in the network [34]. The data categorization and the reserving of slots for the transfer of the data to the sink node are carried out in accordance with the severity level of the data. The sequential procedures in the MSC, such as preprocessing, equivalent class set generation, index estimate, and storage optimization, result in the production of regular and emergency data with the least amount of energy usage possible.

Gomes et al. [35] have recommended the usage of devoted protuberances to screen the connection quality. This technique employs a hybrid channel diversity scheme in which the beacon frames are sent using channel hopping and the unicast data packets are transmitted using channel adaptation. When there are changes in the channel quality across time and space, the network dynamically assigns channels to accommodate these changes.

Li et al. [36] have suggested a cloud-assisted architecture, which consider aspects like quality of service (QoS), handover number, AP loads, and other variables. By recasting the issue as a combinatorial optimization problem and offering a cloud-assisted ant colony-based solution, we have made significant progress (CAFP). AP series are constantly adjusted to meet the goal of decreasing latency and the frequency of handovers while also meeting additional limitations, such as varied handover timings and changing network conditions. In order to further minimize handover time, including handover judgement, the solution makes use of cloud-based support. This has been shown via rigorous simulations and experiments.

According to Wang et al. [37], they have suggested a significant step toward breaking the vicious "break-fixbreak-fix" cycle that has plagued the two-factor authentication research arena for industrial WSNs in recent years. The advantages and disadvantages of eight fundamental system designs are fully defined, as is the widely recognized adversary model, which is used to evaluate a complete set of twelve independent assessment metrics. It is important to note that the sensor nodes are very small and light devices with limited compute performance, storage capacity, and energy resources. Then, they examined two of the most widely utilized schemes in order to identify the problems and obstacles associated with developing a sound system.

A distributed filtering approach for the fault recognition issue using wireless sensor networks (WSNs) has been presented by Gao et al. [24]. When dealing with nonlinear stochastic systems that have a discrete-time form, Takagi-Sugeno (T-S) fuzzy models of interval type 2 (IT2) are used to describe them. Each sensor in the WSN may receive measurements from both itself and its nearby sensors, provided that the sensors are connected in a deterministic interconnection pattern. A set of independent random variables following the Bernoulli distribution is created in order to describe the randomly occurring packet losses between the wireless sensor network and the filter unit. In order to provide residual signals for the evaluation functions of the fault detection mechanism, a unique form of IT2 T-S fuzzy distributed fault detection filter is used in conjunction with a sensor node for each sensor node. It is decided to use a fault reference model in order to improve the performance of the fault detection system, which is implemented as an IT2 T-S fuzzy model.

Using vibration analysis, Lu et al. [38] have presented a system for motor bearing condition monitoring and problem detection that is based on vibration analysis. It consists 
of four steps: determining the resonance band of the oversampled vibration signal using a kurtogram, filtering out the unwanted noises in the vibration signal on an analogue level, bandpass sampling the filtered signal, and applying the demodulated resonance technique for bearing fault detection in the digital domain.

In the context of machine condition monitoring applications, the IWSN has some limitations, including lower throughput due to larger data volumes, target coverage issues, a lower packet delivery ratio due to losses, and higher energy consumption due to the use of battery-operated sensor nodes, among others. A unique routing algorithm and data processing mechanism should be developed in order to achieve energy-efficient and reliable data transfer in the IWSN.

A variety of expert systems are available to identify and categorize the flaws of machines in the fault diagnostic approaches, which are described in more detail below. Despite the fact that current signature analysis, principal compound analysis, and various artificial intelligence methods have all contributed to significant advancements in the field of machine fault diagnosis, there is still some complexity in the mathematical modelling, optimization techniques, learning process, time consistency, and accuracy level that must be addressed. It is necessary to use the simplest and most efficient methods possible in order to achieve early problem identification and isolation in industrial settings.

When compared to the usual oversampling approach, the findings demonstrate that utilizing this method leads to a reduction of data length and transmission time of more than 80 percent. However, it must be noted that before these compression techniques can be used, the signal must be greater than the Nyquist rate, and that these compression methods consume a significant number of computational resources. This approach may be used in conjunction with the usual data density method to lower the amount of data that is stored.

\section{Methodology of the Proposed System}

3.1. Problem Methodology. In the industrial sector, there are hundreds of machinery and pieces of equipment. Individual machine or equipment failure may result in selfinflicted harm as well as financial loss. This is due to the interruption in the flow of an industrial process that happens throughout the manufacturing process. It is vital to monitor the specific machines in the business in order to limit the unwelcome downtime that occurs. The circumstances for machine monitoring are created utilizing the most up-to-date technology. Specific companies employ wired Internet monitoring systems for this reason as well, which may be found in some sectors. For small- and medium-sized businesses, wired Internet monitoring systems are used. However, monitoring key devices on vast industrial sites may be a difficult undertaking to do. It will cost more money to monitor noncritical devices that are not checked on a regular basis when they are paired with a wired monitoring system. It is difficult to make changes to a wired system if there is a momentary shift in the industry's requirements.

In Cai et al.'s work [12], a delay-aware mobile WSN routing algorithm (MWSN) is presented to meet the requirements of current industrial circumstances. To achieve real-time information sharing and better quality of service (QoS) in terms of delay times, the MWSN is built on a cyberphysical system architecture. For the purpose of determining the shortest path for data packet transmission, the classic geographic routing protocol is utilized. When the AGVs are travelling around the production area, this protocol is utilized to determine the shortest path to take. The optimization method may be used to minimize the number of hops required in routing lines. The hop is critical parameter since it has a direct impact on the delay time of the signal. Because of this, fault detection is a key operation in an industrial monitoring system. But it is regarded as a tough and complicated undertaking owing to the existence of elements that might impact data and perhaps generate errors in the process. Fault is regarded to be an application, and the sort of sensor used to detect it varies.

To diagnose the issue, machine learning techniques are often utilized. These approaches are divided into three separate stages, which are feature extraction, feature selection, and classification. It is necessary to choose acceptable objects at each level of machine learning in order to develop a defect diagnostic system that is efficient in terms of computing time. On the basis of the energy efficient delayaware WSN routing (EDR) protocol for fault detection, a new industrial environment monitoring system (IEM) with fault detection is developed. The proposed IEM-EDR protocol consists of different algorithms:

(i) First, the group optimal algorithm used to form the clustering which will reduce the energy consumption of nodes

(ii) Then, introduce the node selection algorithm for the aggregated node computation among multiple nodes in the network

(iii) The modified task selection algorithm is used to compute the optimal path between industrial environments to data processing centers (monitoring room)

3.2. System Model. The suggested system model is shown in Figure 1. There is an industrial environment as part of the system. Three distinct sensors, namely, a temperature sensor, a pressure sensor, and a speed sensor, are utilized to detect the temperature, the pressure, and the speed of the vehicle. The employment of a data monitoring center is necessary for the monitoring of data transfer. There are vehicles that are also referred to as automated guided vehicles (AGVs). The trucks will collect data from the base station and transmit it to nodes in the surrounding area. The terminal ranges are utilized to locate the node that is closest to the terminal ranges. The nodes are grouped together. Transmission pathways are generated between the nodes as a 


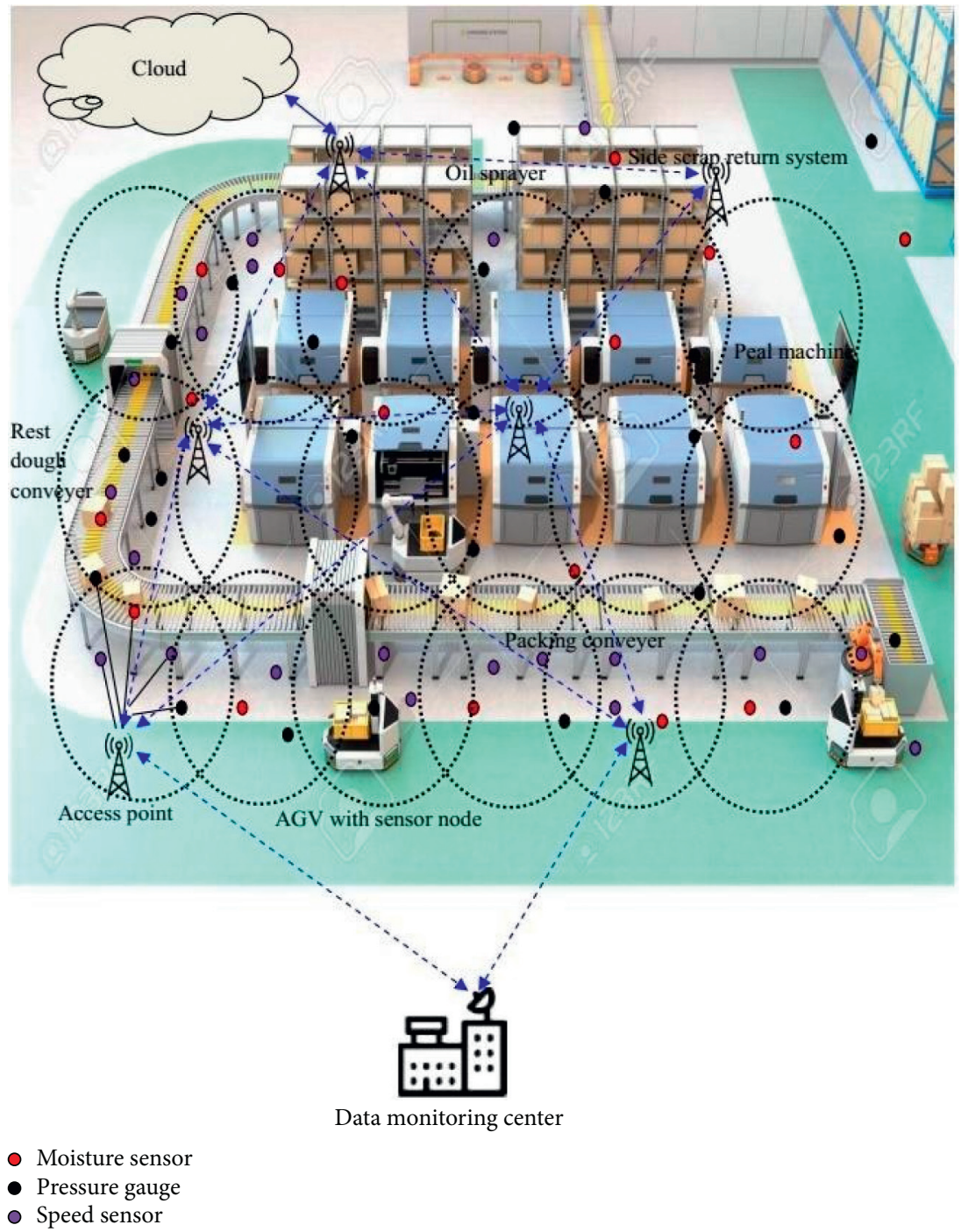

FIGURE 1: System model of the proposed IEM-EDR protocol.

result of the formation of paths. It is possible to identify aggregated nodes from a large number of nodes.

Data transmission is ensured by the presence of an access point or base station. The data that is being sent from the sensors is being sent to the cloud platform, and only after that will it be sent to the monitoring device. In order for the sensor to communicate data to the car, it must first submit a request to the base station, which will then look for a vehicle that is not already in use. The base station will look for a free car and notify the user. The sensor will retain data until the base station sends an acknowledgment indicating that a free vehicle has been identified. If the whole vehicle is in use, the sensor will keep the data in the sensor node. This will cause the internal processes of the industry to be in an interrupted state throughout that period of time.

In the industrial sector, there are hundreds of machineries and pieces of equipment. Individual machine or equipment failure may result in self-inflicted harm as well as financial loss. This is due to the interruption in the flow of an industrial process that happens throughout the manufacturing process. It is vital to monitor the specific machines in the business in order to limit the unwelcome downtime that occurs. The circumstances for machine monitoring are created utilizing the most up-to-date technology. Specific companies employ wired Internet monitoring systems for this reason as well, which may be found in some sectors. For small- and medium-sized businesses, wired Internet monitoring systems are used. However, monitoring key devices on vast industrial sites may be a difficult undertaking to do. It will cost more money to monitor noncritical devices that are not checked on a regular basis when they are paired with a wired monitoring system. It is difficult to make changes to a wired system if there is a momentary shift in the industry's requirements.

\section{Proposed Methodology}

This section describes the proposed protocol. The proposed IEM-EDR consists of three different algorithms for the cluster formation, path selection, and aggregated node selection. 
4.1. Group Optimal Algorithm Using Cuckoos Search for the Cluster Formation. Clustering is defined as the grouping of sensor nodes by bringing them together over a dedicated network connection. Because the sensor nodes in a bigger WSN operate together, it is possible to cluster them based on the operation they are doing. Each cluster has a root node, which is used to organise the cluster's information. The development of clusters has the potential to extend the life of a networking system. The data in the cluster will be collected by the cluster's leader. The data from the base station will be collected by the cluster head, which will then aggregate and transfer the data to the target node. The following are the parameters that were utilized in the clustering process:

Residual Energy. During the clustering process, after a number of rounds have been completed, the selection of the cluster head is made based on the amount of energy that has been retained in the sensors.

Initial Energy. This option is used to determine which cluster head should be selected. It is defined as the amount of energy that the nodes have when the algorithm begins.

Rate of Energy Consumption. The formula for calculating the rate of energy consumption is provided in the following:

$$
E=\frac{E_{i}-E_{\underline{i}}(t)}{\gamma-1} .
$$

Each node's initial energy and residual energy are represented by the symbols $E_{i}$ and $E_{i}(t)$, respectively, while $t$ represents the current round.

Model of Energy Consumption. A significant quantity of energy is used by the sensor nodes, and the equation for this consumption is provided by

$$
E_{t}(m, d)=\left\{\begin{array}{l}
m \cdot E_{\mathrm{elec}}+m \cdot \varepsilon_{f s} \cdot d^{2} d \leq d_{0}, \\
m \cdot E_{\mathrm{elec}}+m \cdot \varepsilon_{m p} \cdot d^{4} d>d_{0} .
\end{array}\right.
$$

At the receiving end the energy consumption mainly depends on the data size and the receiving energy is defined as

$$
E_{r}(m)=\left\{\begin{array}{l}
m \cdot E_{\text {elec }} \text { nodenot intermediate } \\
m \cdot E_{\text {elec }}+m \cdot E_{d a} \text { nodeis intermediate }
\end{array}\right.
$$

$E_{t}$ is the amount of energy wasted per bit sent. $E_{\mathrm{r}}$ is defined as the amount of energy wasted per bit received at the receiver; Eelec is the amount of energy consumed by the electronic circuit in the transmitter and the receiver; and $E_{\text {elec }}$ is the amount of energy consumed by the electronic circuit in the transmitter and the receiver. It is the amount of energy spent in one bit data aggregation that has been calculated. The free space coefficient factor $(f s)$, the multipath coefficient factor $(m p)$, the size of the data packet $(m)$, the threshold distance $(d 0)$, and the distance between the transmitter and the receiver $(d)$ are all defined as follows.
4.2. Cluster Formation Using Group Cuckoos Algorithm. Yang and Deb introduced the optimum Cuckoos Search method in 2009 [39], which has now been implemented. Based on the obligatory brood parasitism of certain cuckoo species, which involves depositing their eggs in the nests of other host birds of other species, it is a viable strategy. It is necessary to apply the CS to solve the maximising issue in multidimensional space. The value of the objective function determines the quality or fitness value of the objective function. The following are the three rules that govern the CS:

It only lays one egg at a time and deposits its eggs in a randomly selected nest. The next generation is estimated by selecting the best nests that produce high-quality eggs.

When it is assumed that there are a set number of host's nests, the likelihood of a cuckoo laying an egg is computed, and the probability is denoted by the letter $P$. The worst nests are detected as a result of this.

The fitness values are determined in various ways depending on the solutions chosen. By incorporating the product of the levy angle and step size into the previous solution, the new solution may be updated to move closer to the ideal point in the optimization process. The new solution for the cuckoo is provided by $X$. The levying formula is described in the next section:

$$
\begin{aligned}
& X_{i}^{t+1}=X_{i}^{t}+\alpha \operatorname{Levy}(\widetilde{n}), \\
& X_{i}^{t+1}=X_{i}^{t}+\alpha * E_{t},
\end{aligned}
$$

where $E_{t}$ is taken from a standard normal distribution and it has zero mean and unity standard deviation for random walks, or drawn from Levy distribution for Levy flights. The step size is given by $\alpha$ and its value is greater than zero (normally it is taken as 1) (Algorithm 1).

\subsection{Modified Task Selection Algorithm Used to Compute the} Optimal Path. The main aim of the algorithm is to identify the optimal path between the nodes. The links are formed using the chemical reaction algorithm.

\subsection{Modified Task Selection Algorithm Using Chemical} Reaction Optimization. The chemical reaction optimization (CRO) is based on swarm intelligence metaheuristics, which are used to find the best solution. The molecular structure found in the CRO provides a comprehensive answer. It is generally accepted that molecules contain two kinds of energies, namely, potential energy (PE) and kinetic energy (KE), which are typically found in equal amounts (KE). A stable PE is one that is characterized by the virtue of its structure, which may be described as follows: If the PE is low, this indicates that the structure is stable. However, in KE, the energy held by the molecule as a result of its motion is considered. The potential energy function of a molecule may 
Input $\mathrm{x}_{\mathrm{i}}$ and value of $\mathrm{i}$.

Output Cluster formation

(1) Objective function is described

(2) Initialize the population of $n$ host is $\mathrm{x}_{\mathrm{i}}(i=1,2, \& \ldots n)$

(3) if $(\mathrm{t}<\mathrm{Maxg})$

(4) Calculate the step size by: Step size $=r$ and $*(\operatorname{nest}(\operatorname{randperm}(n),:)-\operatorname{nest}(\operatorname{randperm}(n))$,$) ; new_nest =$ nest + stepsize. * $K(6)$ where $K=\operatorname{rand}(\operatorname{size}($ nest $))>$ pa.

(5) Select a cuckoo randomly based on the levy flights and also calculate the fitness value $f_{i}=\left(\left(\sum_{i=1}^{n} F \times w_{i}\right) \times 100 / l\right)$ where $F-i=1$ frequency, $w$ is the weight and $l$ is the message length.

(6) Select nest among $n$ host

(7) if $\left(f_{i}>f_{j}\right)$

(8) Worse nest is assigned as $P_{\mathrm{a}}$ and new nest are identified.

(9) Maintain the best solution

(10) Repeat up to the best position is obtained.

(11) End

Return Cluster Formation

Algorithm 1: Group cuckoos algorithm for clustering formation.

be measured, and the quality of a molecule can be estimated. $\mathrm{PE}$ may be calculated using the following formula:

$$
\mathrm{PE}_{\chi}=\mathbf{f}(\chi)
$$

The change in the molecular structure is from $\chi$ to $\chi^{\prime}$ and it is defined as $\mathbf{P E}_{\chi} \geq \mathbf{P E}_{\chi^{\prime}}$ or it can also be defined as $P E_{\chi}+K E_{\chi} \geq P E_{\chi^{\prime}}$. The capacity of the molecules to escape from a local optimum is defined by their KE value. A distinction is made between unimolecular collisions and intermolecular collisions when it comes to molecule-tomolecule collisions. The collision of a single molecule is subdivided into two types: on-wall ineffectual collision and disintegration. Ineffective collision and synthesis are the two categories of intermolecular interaction. The mathematical expression is represented as follows.

On-Wall Ineffective Collision. The expression is given by

$$
\mathrm{PE}_{\chi}+\mathrm{KE}_{\chi} \geq \mathrm{PE}_{\chi^{\prime}}
$$

Here, $\chi$ is the current molecular structure; the changed structure is given by $\chi^{\prime}$.

However, the effect is not as dramatic as it may be. The creation of the following particle is not dissimilar from that of the initial particle, and the equation for the subsequent particle is

$$
\mathrm{KE}_{\chi^{\prime}}=\mathrm{PE}_{\chi}+\mathrm{KE}_{\chi^{\prime}}-\mathrm{PE}_{\chi^{\prime}} \times \mathbf{p}, \mathbf{p}\left[\mathrm{KE}_{\mathrm{LR},}\right] \text {, }
$$

where $\mathrm{KE}_{\mathrm{LR}}$ is the organization limit restricted by $[0,1]$ and the quantity of energy lost to the environment when a molecule hit is given by

$$
\mathrm{PE}_{\chi}+\mathrm{KE}_{\chi}-\mathrm{PE}_{\chi^{\prime}} \times(1-\mathbf{p})
$$

The energy that has been lost is stored in the central energy buffer. The energy that has been stored may be utilized to further the decomposition process.

Decomposition. After hitting with the wall of the container if the molecules splits into two or more pieces, it is known as decomposition. The collision is energetic; the structure of the particles is difficult to obtain. The condition is given by

$$
\mathbf{N}_{\mathbf{H}}[\chi]-\mathbf{N}_{\mathbf{H}}[\chi]>a .
$$

The original molecule structure is $\chi$ and the obtained structures are $\chi_{1}^{\prime}$ and $\chi_{2}^{\prime}$. The changed structure is given by

$$
\mathrm{PE}_{\chi}+\mathrm{KE}_{\chi} \geq \mathrm{PE}_{\chi_{1}^{\prime}}+\mathrm{PE}_{\chi_{2}^{\prime}} \text {. }
$$

The value is assigned as

$$
\operatorname{temp}_{1}=P E_{\chi}+K E_{\chi} \geq P E_{\chi_{1}^{\prime}}+P E_{\chi_{2}^{\prime}} .
$$

Therefore,

$$
\begin{aligned}
K E_{\chi_{1}^{\prime}} & =\operatorname{temp}_{1} \times q K E_{\chi_{2}^{\prime}} \\
& =\operatorname{temp}_{1} \times(1-q),
\end{aligned}
$$

where $q$ indicates the random number uniformly generated from the interval $[0,1]$. Commonly, $P E_{\chi^{\prime}} P E_{\chi_{1}^{\prime}}$ and $P E_{\chi_{2}^{\prime}}$ have similar energy. Due to a sequence of on-wall unproductive collisions, the KE of molecules decreases. Thus, from the central energy, some energy is drawn; this is used for the decomposition reaction:

$$
\mathrm{PE}_{\chi}+\mathrm{KE}_{\chi}+\text { buffer } \geq \mathrm{PE}_{\chi_{1}^{\prime}}+\mathrm{PE}_{\chi_{2}^{\prime}} .
$$

If (14) holds, the change is allowed, and we can calculate

$$
\begin{aligned}
& K E_{\chi_{1}^{\prime}}=\left(\text { temp } p_{1}+\text { buffer }\right) \times q_{1} \times q_{2}, \\
& K E_{\chi_{2}^{\prime}}=\left(\text { temp }_{1}+\text { buffer }-K E_{\chi_{1}^{\prime}}\right) \times q_{3} \times q_{4} .
\end{aligned}
$$

The random uniform numbers are indicated using $q_{1}, q_{2}$, $q_{3}$, and $q_{4}$ and the random numbers are generated from the interval $[0,1]$. The buffer is updated as

$$
\text { buffer }=\text { buffer }+ \text { temp } p_{1}-K E_{\chi_{1}^{\prime}}-K E_{\chi_{2}^{\prime}} \text {. }
$$

Intermolecular Ineffective Collision. If more molecules collide each other and after that they bounce back, this 
collision is similar to on-wall ineffective collision. Form the central energy buffer, no KE is drawn. Here, the only exchange of energy takes place between the molecules.

The initial position of the molecules is given by $\chi_{1}$ and $\chi_{2}$. The obtained structure is taken as $\chi_{1}^{\prime}$ and $\chi_{2}^{\prime}$ where $\chi_{1}^{\prime}$ and $\chi_{2}^{\prime}$ are neighborhood structures. The exchange will happen only when the conservation of energy condition holds.

$$
\mathrm{PE}_{\chi_{1}}+\mathrm{KE}_{\chi_{1}}+\mathrm{PE}_{\chi_{2}}+\mathrm{KE}_{\chi_{2}} \geq \mathrm{PE}_{\chi_{1}^{\prime}}+\mathrm{PE}_{\chi_{2}^{\prime}}
$$

It is assigned to a variable and is given by

$$
\text { temp } \mathbf{p}_{2}=\mathrm{PE}_{\chi_{1}}+\mathrm{KE}_{\chi_{1}}+\mathrm{PE}_{\chi_{2}}+\mathrm{KE}_{\chi_{2}}-\mathrm{PE}_{\chi_{2}^{\prime}}+\mathrm{PE}_{\chi_{2}^{\prime}} \text {. }
$$

Therefore,

$$
\begin{aligned}
& K E_{\chi_{1}^{\prime}}=\operatorname{temp}_{2} \times r, \\
& K E_{\chi_{2}^{\prime}}=\operatorname{temp}_{2} \times(1-r) .
\end{aligned}
$$

The random uniform number is generated between the interval $[0,1]$.

Synthesis. The collision of the molecule occurs, and a middle particle or novel particle is formed. The collision is energetic. From the changed construction, it is very difficult to get the original molecule structure. The condition is given by

$$
\begin{aligned}
& K E_{\chi_{1}} \leq \beta, \\
& K E_{\chi_{2}} \leq \beta .
\end{aligned}
$$

Suppose the original molecules are $\chi_{1}$ and $\chi_{2}$; the obtained molecule is $\chi^{\prime}$. The condition is given by

$$
\begin{gathered}
\mathbf{Q F}_{\chi_{1}}+\mathbf{L F}_{\chi_{1}}+\mathbf{Q F}_{\chi_{2}}+\mathbf{L F}_{\chi_{2}} \geq \mathbf{Q} F_{\chi^{\prime}} \\
L F_{\chi^{\prime}}=Q F_{\chi_{1}}+L F_{\chi_{1}}+Q F_{\chi_{2}}+L F_{\chi_{1}}-Q F_{\chi^{\prime}} .
\end{gathered}
$$

Energy Model. It is dependent on the quantity of data to be sent as well as the distance to be travelled. If the propagation distance $(d)$ is less than the threshold distance $(d 0)$, the energy consumption is proportional to the square of the distance $(d 2)$. Unless otherwise stated, $d 2$ is proportional to $d 4$. The entire amount of energy used by each node is represented by

$$
F_{U Y}(m, e)=\left\{\begin{array}{l}
e \times F_{\mathrm{elec}}+\varepsilon_{g t} \times e^{2}, i f e<e_{0}, \\
e \times F_{\mathrm{elec}}+\varepsilon_{n q} \times e^{2}, i f e \geq e_{0},
\end{array}\right.
$$

where $E_{\text {elec }}$ is the energy degenerate per bit when the spreader or handset circuit is operating.

$\varepsilon_{f s}$ indicates the amplifier energy in the free space; $\varepsilon_{m p}$ is contingent on the spreader amp perfect and the edge broadcast coldness. The energy consumed by the receiver is given by Algorithm 2:

$$
\mathrm{E}(\mathbf{l})=\mathbf{l} \times \mathrm{E}_{\mathrm{elec}}
$$

4.5. Node Selection Algorithm for the Aggregated Node. The selection of the aggregated node is accomplished via the usage of node selection. The method that was employed in the selection of the weed optimization algorithm was described before. The next section is a more in-depth examination of the weed algorithm.

The weed optimization algorithm is used in the search for the aggregated node. It is either based on the WOA's agricultural format or on the weed's growth characteristics, depending on which is most appropriate. Normally, weed plants sprout up out of nowhere and cause damage to pastures, farms, and vegetable crops. It may be utilized in any location and under all circumstances. Many characteristics of plants, such as seed competition, production, and growth, are statistically replicated by the WOA. The following criteria are necessary for the growth of weed colonies:

(i) Only a limited number of seeds are disseminated in each region

(ii) Depending on the quality of the colony, the seed develops into a weed that produces seeds again

(iii) The seeds that have been created will grow in a random manner in the designated area and will produce additional seeds

(iv) The process will continue until the colony has achieved its maximum number of plants

Weeds of lesser quality are eradicated based on the results of the analysis. Because of this, weeds of high grade will be selected for cultivation. The following is the first step in WOA.

Assigning the initial population: the original population is represented by the following: in the beginning, the population is produced and spreads out randomly throughout a $d$-dimensional region. The $d$-dimension of the plant is considered as the plant's physical size. The presence of a plant in the region is taken into consideration while making a choice. As a result, a colony will be formed by a group of multiple plants.

Reproduction. As the name implies, the plants in this part will reproduce in order to produce seed that will be used to establish the colony. The seeds are created by selecting their own quality, the quality of the colony, and the maximum and minimum number of seeds that will be produced. This is represented by the variables (No $S_{\max }$ ) and (No $\left.S_{\min }\right)$, and the user may specify the values that are used to signify this. The following is the expression for the number of speeds:

$$
N_{s}=\frac{F_{i}-F_{\text {worst }}}{F_{\text {best }}-F_{\text {worst }}}\left(N_{\max }-N \min _{\text {min }} .\right.
$$

The adoption and randomization of seeds are the two most important activities that take place during this stage of the process. The produced seeds are randomly distributed in a $d$-dimensional region with a normal distribution and a zero mean when they are created. As a result, a novel situation will emerge in which young plants will develop 
Input $T_{r}$, structure of molecule.

Output Optimal path selection

(1) Division of Transmitter $\left(T_{r}\right)$ into one molecular and two molecular structures.

(2) Case 1: One molecular structure

(3) if (Satisfy the decomposition)

(4) Perform Decomposition operation

(5) Else

(6) Perform On-wall ineffective collision.

(7) Case 2: Two molecular structure

(8) if (Satisfy the decomposition)

(9) Perform Synthesis

(10) Else

(11) Perform inter-molecular ineffective collision

(12) For both case 1 and case 2, Check for the new minimum PE.

(13) Repeat the process until optimal solution is obtained.

(12) End

Return Optimal path selection

Algorithm 2: CRO for computing the optimal path.

around the parent plant in three-dimensional space. The standard deviation of the freshly generated plants is quite varied. In the equation, the standard deviation is represented as a percentage (29). It is obvious from the equation that the initial predefined value (maximum) leads to a final predetermined value (minimum) (Algorithm 3).

$$
\sigma_{\text {iter }}=\frac{\left(\text { itern }_{\max }\right.}{\left(\text { itern }_{\max }\left(\sigma_{\text {inital }}-\sigma_{\text {finial }}\right)+\sigma_{\text {finial }}\right.} .
$$

\section{Experimental Results}

The simulations are carried out with the help of the NS2 simulation environment. The simulations are done out using metrics that are applicable to both the proposed protocol and the current protocol. Clearly, when comparing the present MWSN to the proposed system IEM-EDR, it is evident that the existing MWSN has poor performance. The IEM-EDR method, which is comprised of three separate techniques, is suggested in this paper: cluster formation is accomplished by the employment of a group optimum algorithm based on cuckoo search; this reduces the energy consumption of the nodes. The second technique, which makes use of a modified job scheduling algorithm based on the chemical process, is employed for optimum route selection. The third approach is used to pick the aggregated node from a pool of nodes in the network, and it is described below.

For the purposes of this research, the automated guided vehicles (AGVs) and the terminal range are considered two different scenarios. It is possible to determine metrics such as accuracy, latency, fault, loss, and throughput by comparing two cases. The AGVs are believed to be autonomous vehicles that are used to transport data. It is necessary to know the terminal range in order to determine the closest routing point.

The parameters that are often utilized in simulations are taken into consideration, and they are presented in Table 1.
The number of nodes is selected from a range of 10 to 100 . The area is assumed to be 1000500 square feet. The $18720 \mathrm{~J}$ is considered as the beginning energy for each node in the network. The transmission distance is 50 meters.

5.1. Evaluation with $A G V s$. The simulations are carried out with the help of the NS2 simulation environment. The simulations are done using metrics that are applicable to both the proposed protocol and the current protocol. Clearly, when comparing the present MWSN to the proposed system IEM-EDR, it is evident that the existing MWSN has poor performance. The IEM-EDR method, which is comprised of three separate techniques, is suggested in this paper: cluster formation is accomplished by the employment of a group optimum algorithm based on cuckoo search; this reduces the energy consumption of the nodes. The second technique, which makes use of a modified job scheduling algorithm based on the chemical process, is employed for optimum route selection. The third approach is used to pick the aggregated node from a pool of nodes in the network, and it is described below.

For the purposes of this research, the automated guided vehicles (AGVs) and the terminal range are considered two different scenarios. It is possible to determine metrics such as accuracy, latency, fault, loss, and throughput by comparing two cases. The AGVs are believed to be autonomous vehicles that are used to transport data. It is necessary to know the terminal range in order to determine the closest routing point.

The parameters that are often utilized in simulations are taken into consideration, and they are presented in Table 1. The number of nodes is selected from a range of 10 to 100 . The area is assumed to be 1000500 square feet. The $18720 \mathrm{~J}$ is considered as the beginning energy for each node in the network. The transmission distance is 50 meters. Figure 2 gives the accuracy rate. Figure 3 gives the delay rate of the data collected from the machine to the cloud environment. Figure 4 gives the fault rate of the data collected from the machine to the cloud environment. 
Input Population of plants

Output Aggregated node selection

(1) Initial population of the weed

(2) Calculate the fitness value of the initial weed population $\mathrm{F}=\mathrm{af}_{\mathrm{NU}}+\mathrm{bf}_{\mathrm{SLA}}+\mathrm{cf}_{\mathrm{MSL}}$

(3) Calculate number of seeds. Calculate fitness value for each seeds.

(4) Spread the new seeds

(5) Calculate the fitness value for new seed

(6) if (Ns $<$ No_S $\max$ )

(7) Remove the seeds with lower fitness value

(8) else

(9) Maximum number of iterations is achieved

(10) End

Return Aggregated node selection

Algorithm 3: The weed optimization algorithm for the aggregated node.

TABLE 1: Simulation parameters.

\begin{tabular}{lc}
\hline Parameter & Values \\
\hline Number of nodes & 10 to 100 \\
Area & $1000 * 500$ \\
Transmission range & $50 \mathrm{~m}$ \\
Initial battery power & $18720 \mathrm{~J}$ \\
Maximum data pay load size & 1024 bytes \\
Execution time & $65 \mathrm{~s}$ \\
Simulation time & $1000 \mathrm{~s}$ \\
BW & $2.4 \mathrm{GHz}$ \\
Data rate & $250 \mathrm{kbps}$ \\
Packet size & $97 \mathrm{bytes}$ \\
\hline
\end{tabular}

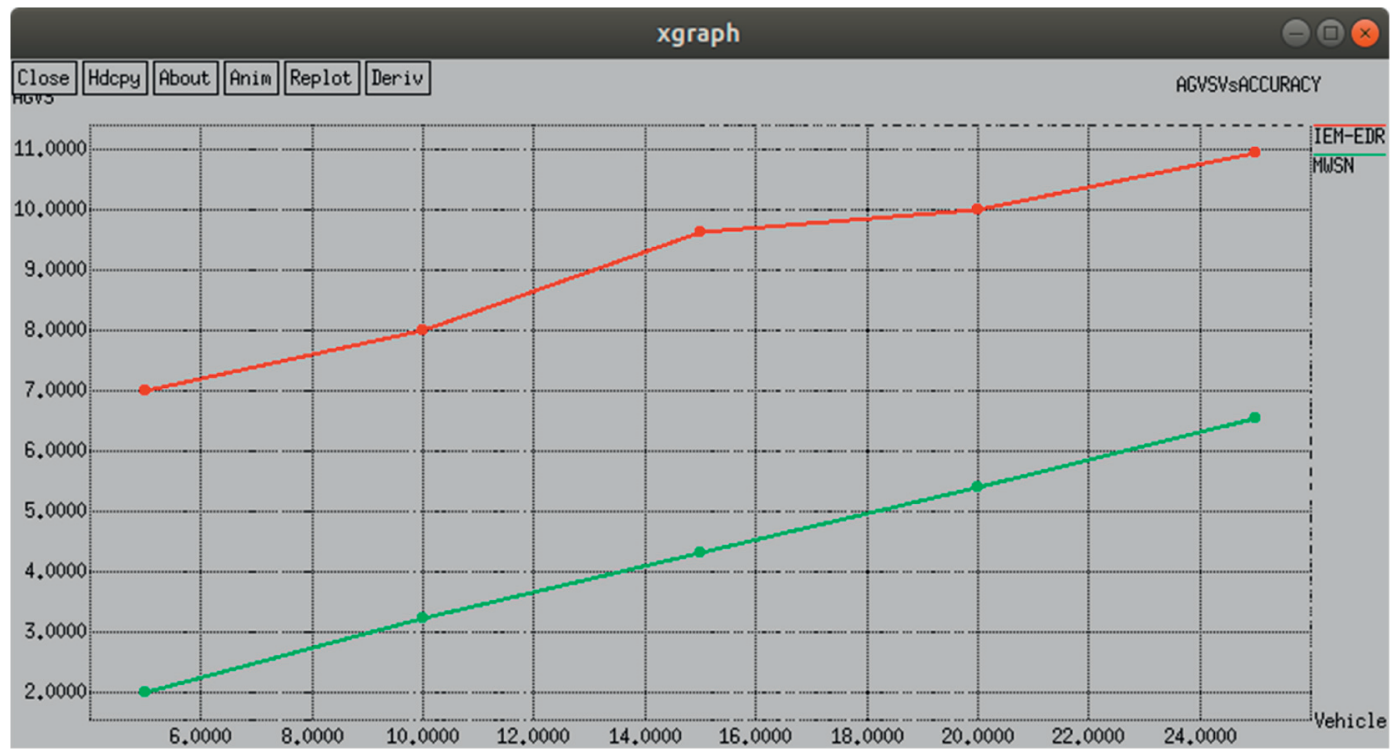

Figure 2: Accuracy with AGVs.

Figure 5 gives the loss rate of the data collected. Figure 6 gives the throughput of the data collected from the machine.

QoS sends data from one node to another in a store and forward manner (also known as hop-by-hop configuration) (also known as hop-by-hop configuration). This sort of architecture enables the data to be reliably transported from source to destination even if the nodes are not linked permanently. QoS rules may be implemented to recognize and priorities RTP (Real-Time Transport Protocol) packets. RTP excels in conveying and processing real-time transmissions of audiovisual data. QoS also enables managers to modify traffic flow on LAN and WAN networks. 


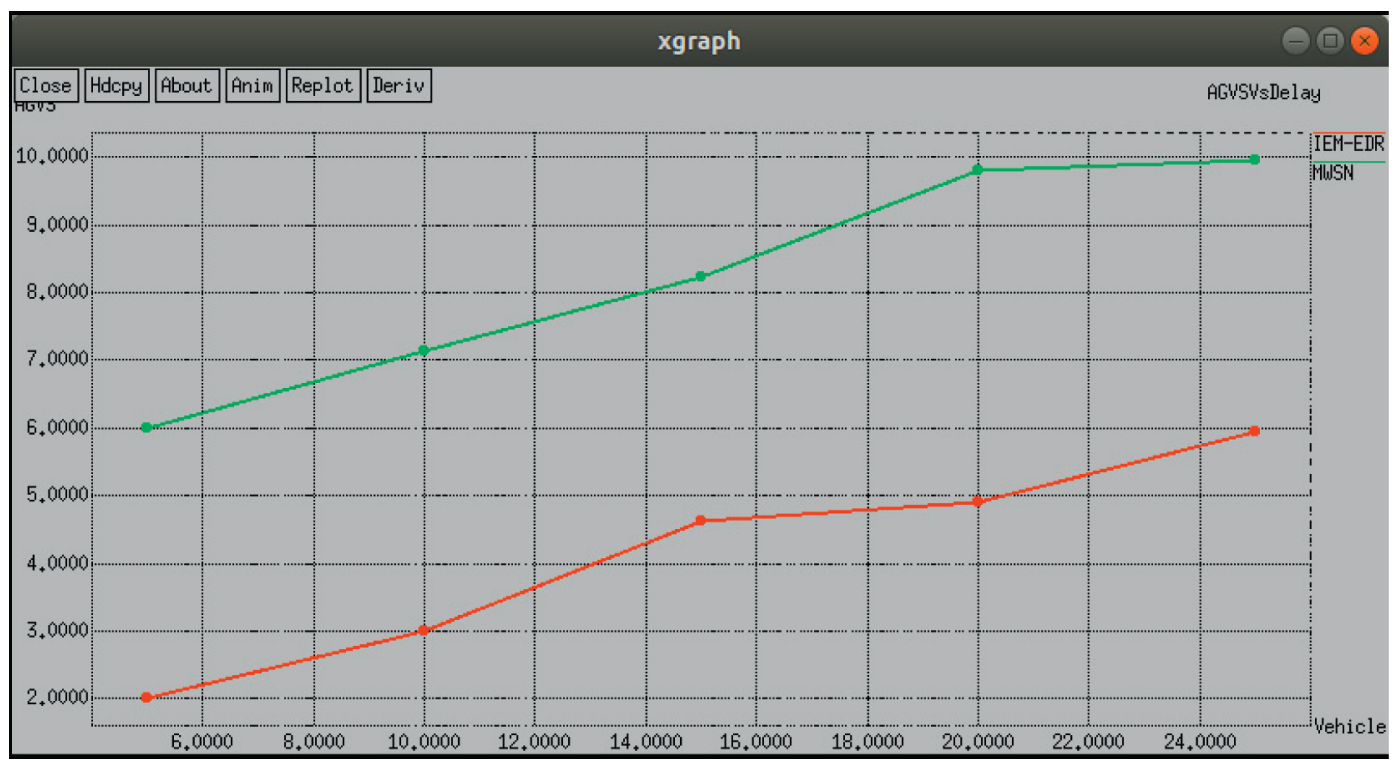

Figure 3: Delay with AGVs.

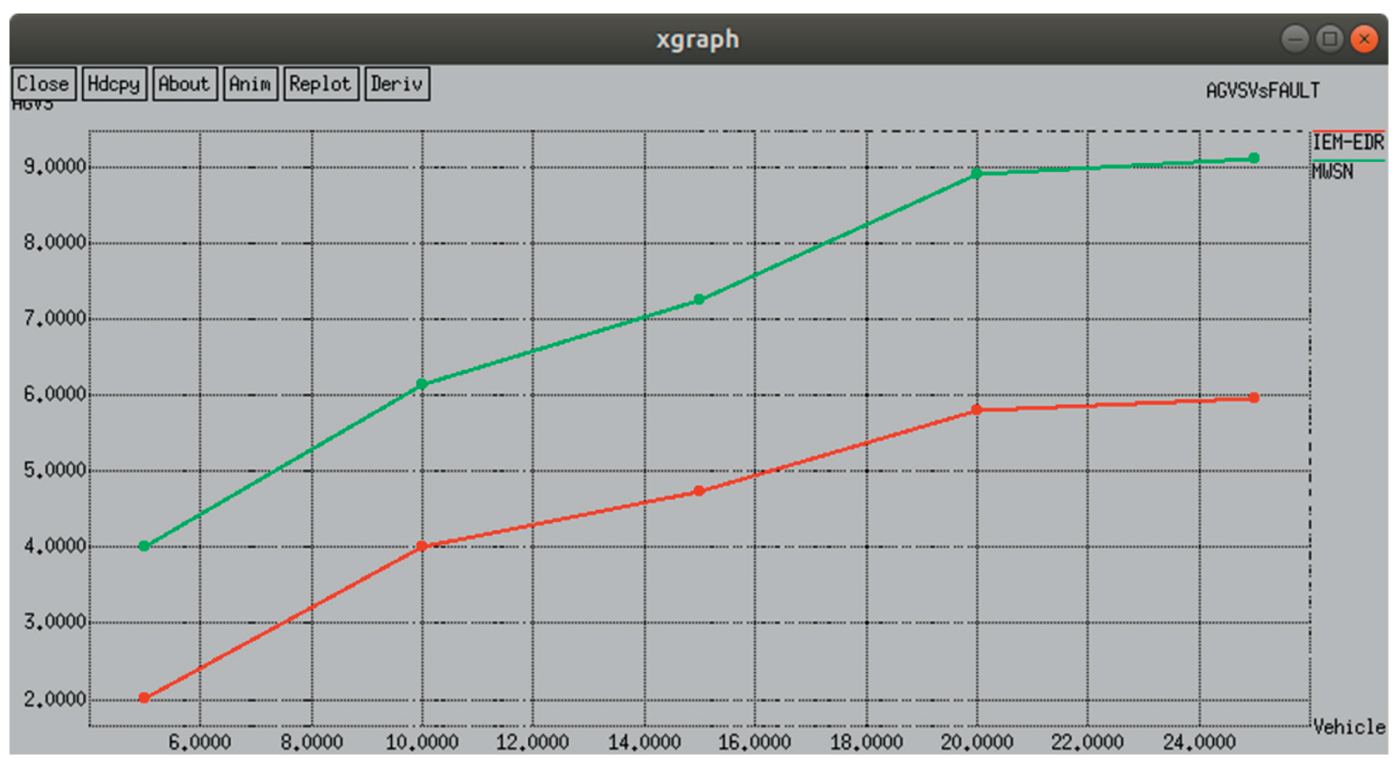

FIgURE 4: Fault with AGVs.

5.2. Evaluation with Terminal Range. Here is a part where you may see the simulation results for the previously indicated parameter with the terminal range. Figure 7 depicts the accuracy analysis in relation to the terminal range. When compared to the current MWSN protocol, the proposed IEM-EDR protocol has a higher level of accuracy. There is a 64 percent increase in accuracy in the IEM-EDR procedure. The delay analysis with the terminal range is shown in Figure 8. It has 62 percent less latency than the present MWSN, according to IEM-EDR results. The error with the terminal range is seen in Figure 9. It is discovered that the IEM-EDR has a 60 percent low fault rate when used. With respect to the terminal range, the loss analysis is shown in
Figure 10. When comparing the IEM-EDR protocol to the present MWSN protocol, it is discovered that the loss rate is reduced by 62 percent. The throughput analysis with a terminal range is shown in Figure 11. In this study, it was discovered that the suggested IEM-EDR protocol would result in a $60 \%$ improvement in throughput.

Figure 8 represents the delay at the terminal range. Figure 9 represents the fault of the terminal range of the input node.

Figure 10 represents the loss of the terminal range in input node.

Figure 11 represents the throughput of the terminal range in input node. 


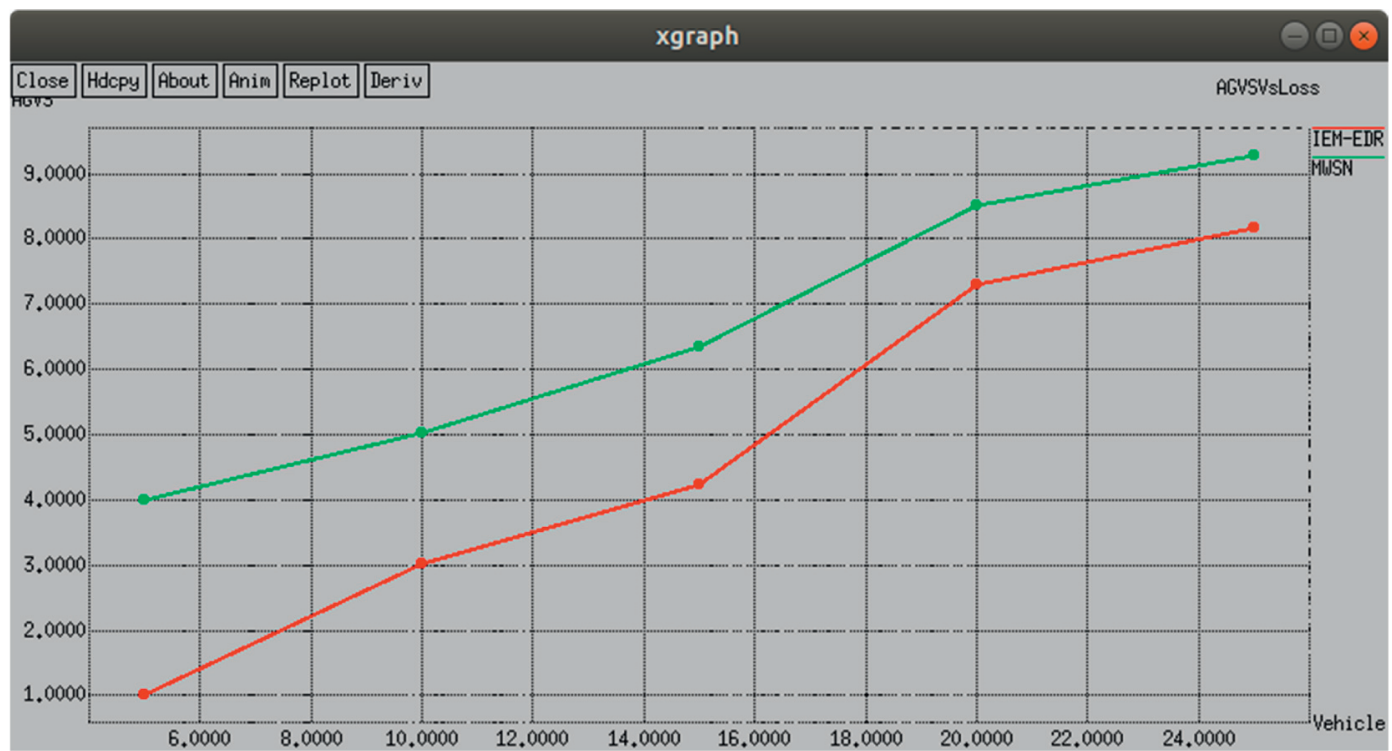

Figure 5: Loss with AGVs.

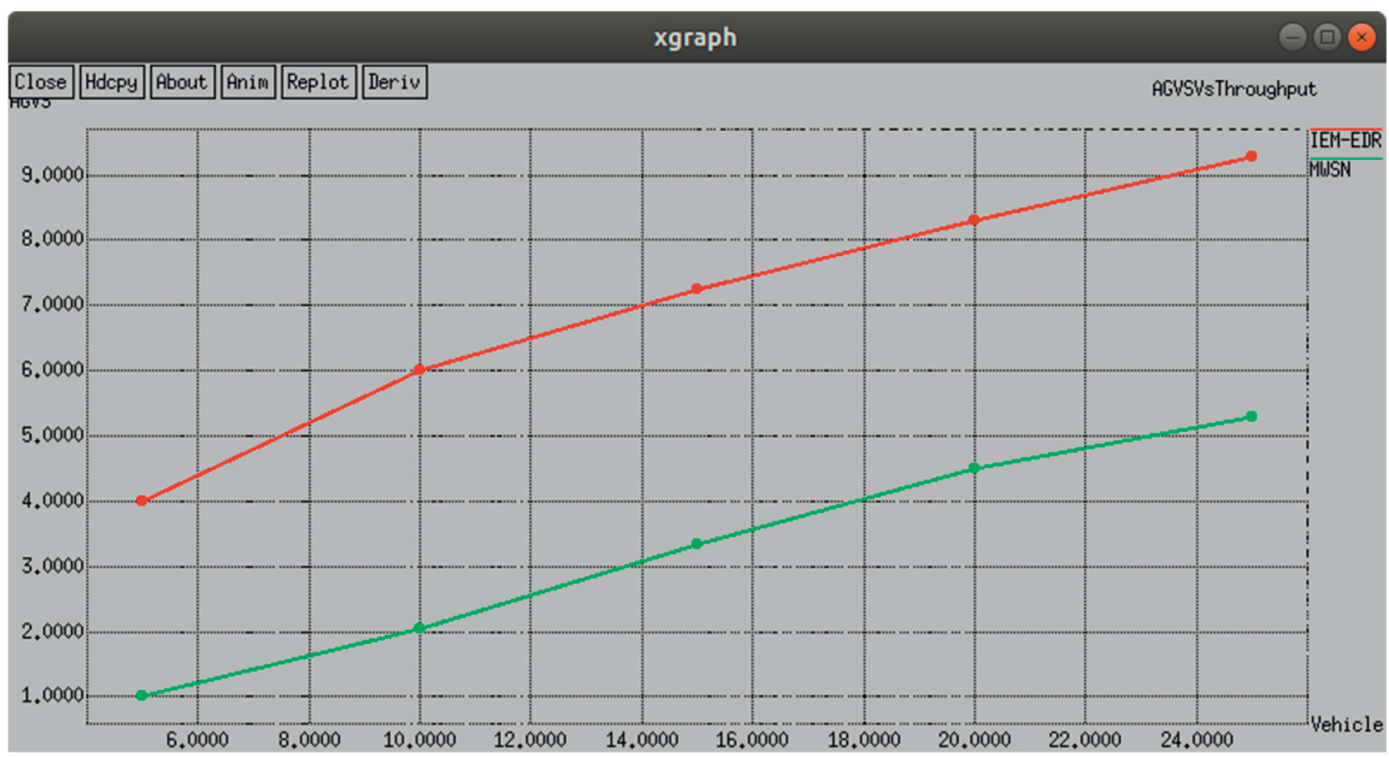

Figure 6: Throughput with AGVs. 


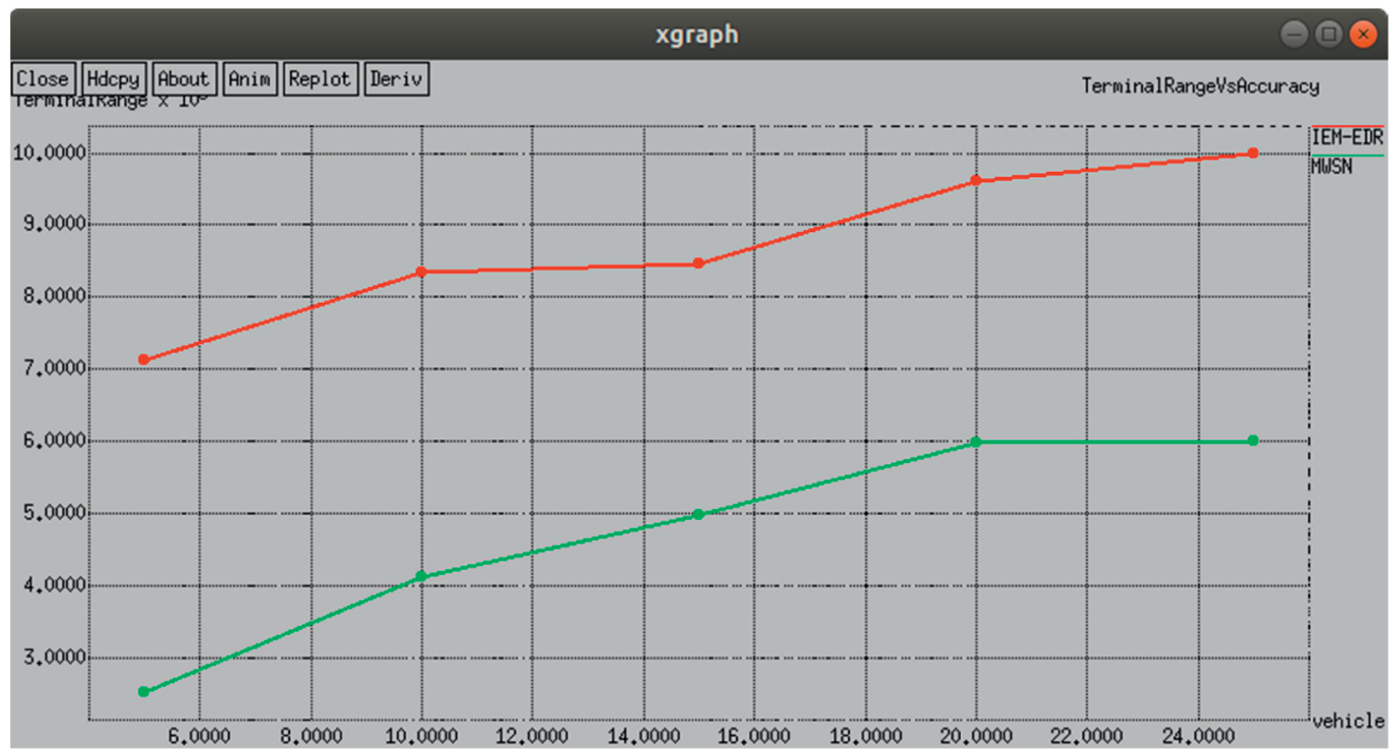

FIgure 7: Accuracy with terminal range.

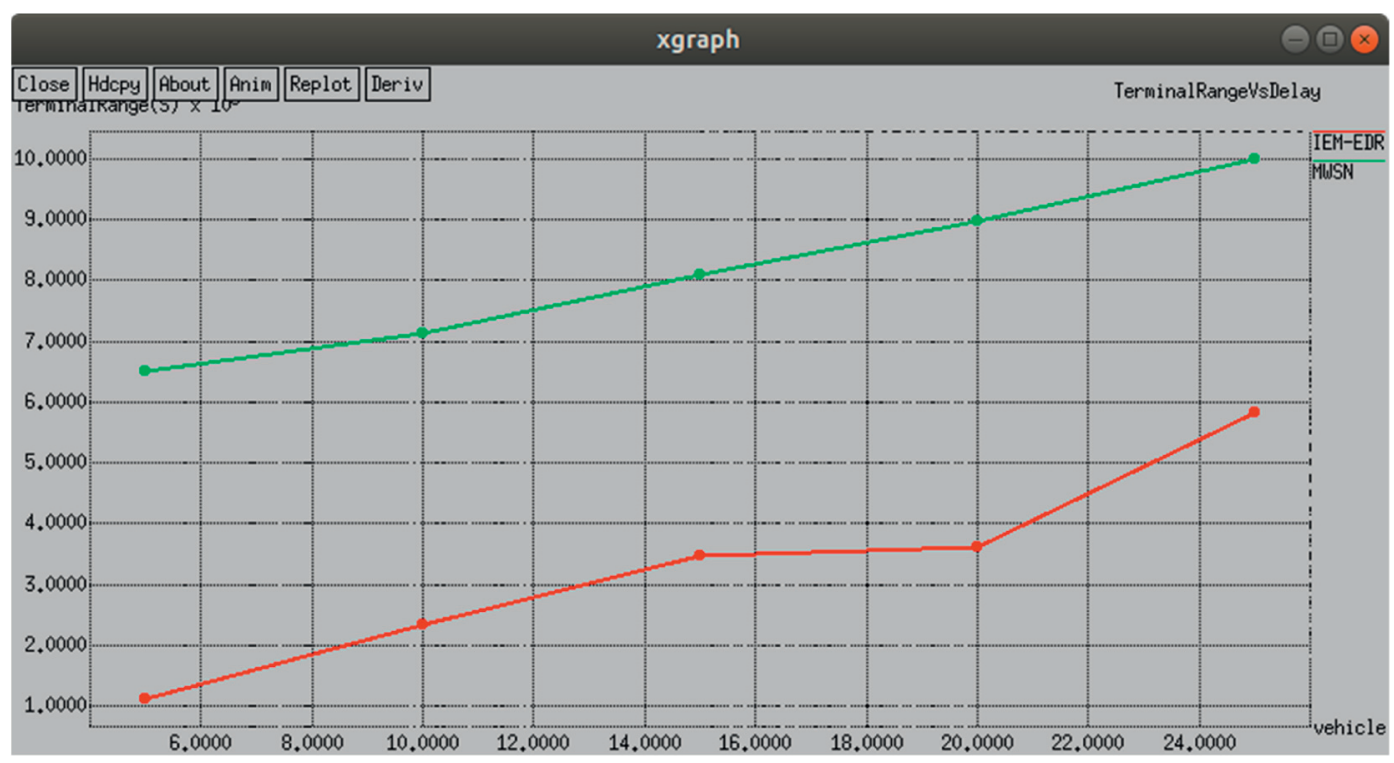

Figure 8: Delay with terminal range. 


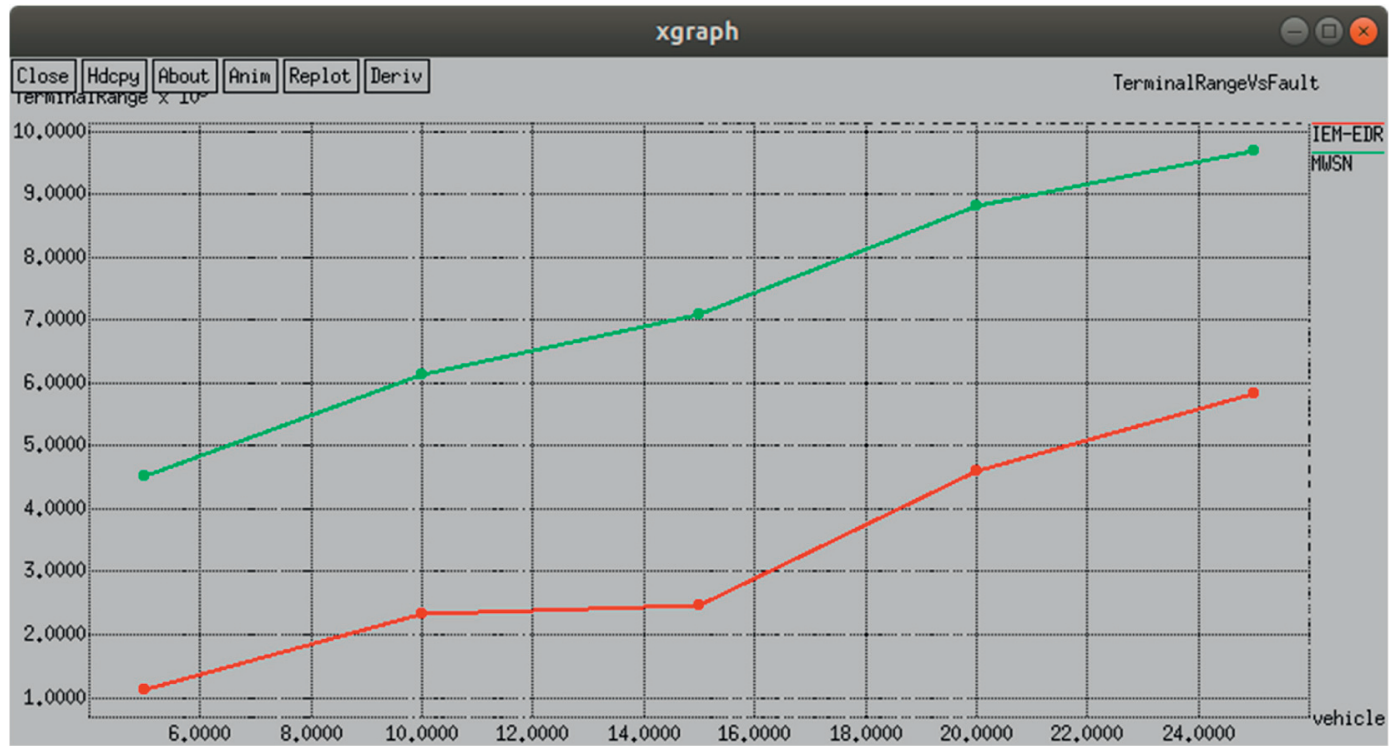

Figure 9: Fault with terminal range.

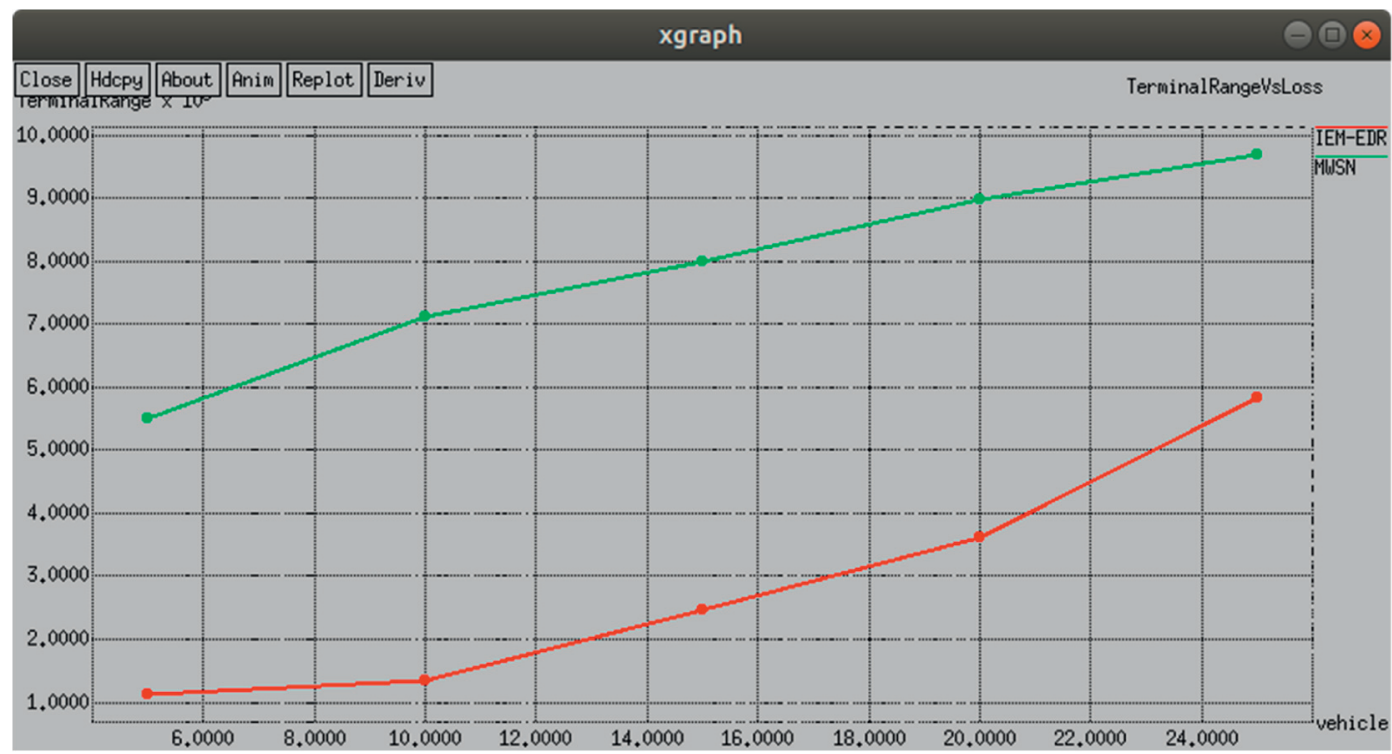

FIgURE 10: Loss with terminal range. 


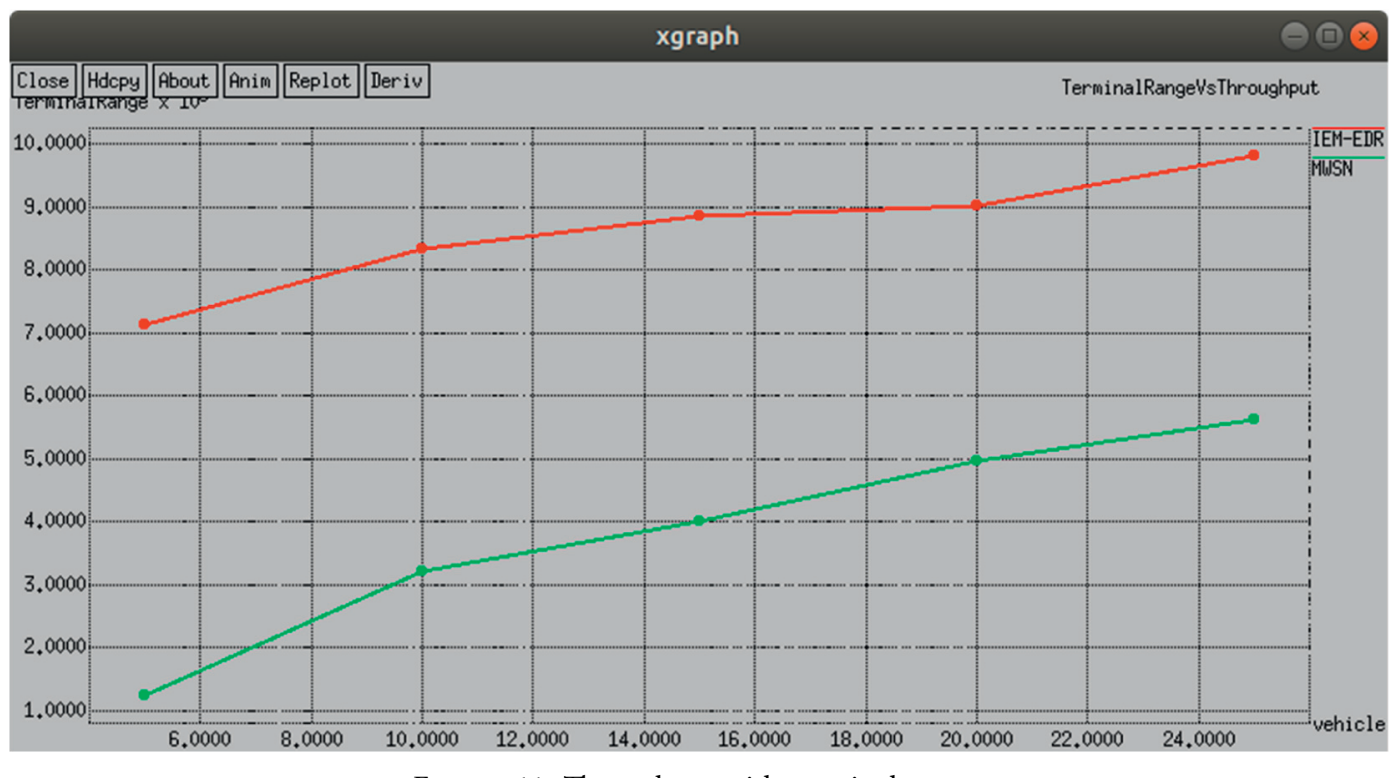

\section{Conclusion}

In contemporary industrial applications, it is critical thing to view equipment and devices besides monitoring and collecting the generated data. This is essential in order to improve the efficiency of production, the security of gadgets, and the competitiveness of the industry. WSN is used for data transmission, control instructions, communications between devices, and other functions. This work describes a new IEM-EDR protocol for the IWSN. The simulation was carried out with the help of NS2. From the results of this study, it is evident that the suggested IEM- EDR protocol has superior performance, when compared to the current MWSN methodology. The forecast of a defect in the machine, based on QoS factors, has an accuracy rate of 95 percent and causes the least amount of data loss possible.

\section{Data Availability}

The data that support the findings of this study are available on request to the author.

\section{Conflicts of Interest}

The author declares that there are no conflicts of interest regarding the publication of this paper.

\section{References}

[1] M. Zhao, J. Li, and Y. Yang, "A framework of joint mobile energy replenishment and data gathering in wireless rechargeable sensor networks," IEEE Transactions on Mobile Computing, vol. 13, no. 12, pp. 2689-2705, 2014.

[2] H. Kim and S.-w. Han, "An efficient sensor deployment scheme for large-scale wireless sensor networks," IEEE Communications Letters, vol. 19, no. 1, pp. 98-101, 2015.

[3] M. Bertocco, G. Gamba, A. Sona, and S. Vitturi, "Experimental characterization of wireless sensor networks for industrial applications," IEEE Transactions on Instrumentation and Measurement, vol. 57, no. 8, pp. 1537-1546, 2008.

[4] F. Salvadori, M. D. Campos, P. S. Sausen et al., "Monitoring in industrial systems using wireless sensor network with dynamic power management," IEEE Transactions on Instrumentation and Measurement, vol. 58, no. 9, pp. 3104-3111, 2009.

[5] W. Xia, R. Neware, S. D. Kumar, D. A. Karras, and A. Rizwan, "An optimization technique for intrusion detection of industrial control network vulnerabilities based on BP neural network," Int J Syst Assur Eng Manag, 2022.

[6] P. Park, C. Fischione, A. Bonivento, K. H. Johansson, and A. Sangiovanni-Vincent, "Breath: an adaptive protocol for industrial control applications using wireless sensor networks," IEEE Transactions on Mobile Computing, vol. 10, no. 6, pp. 821-838, 2011.

[7] L. Palopoli, R. Passerone, and T. Rizano, "Scalable offline optimization of industrial wireless sensor networks," IEEE Transactions on Industrial Informatics, vol. 7, no. 2, pp. 328-339, 2011.

[8] E. Toscano and L. L. Bello, "Multichannel superframe scheduling for IEEE 802.15.4 industrial wireless sensor networks," IEEE Transactions on Industrial Informatics, vol. 8, no. 2, pp. 337-350, 2012.

[9] Y. Ding and S. H. Hong, "CFP scheduling for real-time service and energy efficiency in the industrial applications of IEEE 802.15.4," Journal of Communications and Networks, vol. 15, no. 1, pp. 87-101, 2013.

[10] J. Figueiredo and J. Sá Da Costa, "A SCADA system for energy management in intelligent buildings," Energy and Buildings, vol. 49, pp. 85-98, 2012.

[11] S. Huda, J. Yearwood, M. M. Hassan, and A. Almogren, "Securing the operations in SCADA-IoT platform based industrial control system using ensemble of deep belief networks," Applied Soft Computing, vol. 71, pp. 66-77, 2018.

[12] H. Cai, Y. Zhang, H. Yan, F. Shen, K. Zhou, and C. Zhang, "A delay-aware wireless sensor network routing protocol for industrial applications," Mobile Networks and Applications, vol. 21, no. 5, pp. 879-889, 2016.

[13] H. Kumarage, I. Khalil, Z. Tari, and A. Zomaya, "Distributed anomaly detection for industrial wireless sensor networks 
based on fuzzy data modelling," Journal of Parallel and Distributed Computing, vol. 73, no. 6, pp. 790-806, 2013.

[14] P. Giménez, B. Molina, J. Calvo-Gallego, M. Esteve, and C. E. Palau, "I3WSN: industrial intelligent wireless sensor networks for indoor environments," Computers in Industry, vol. 65, no. 1, pp. 187-199, 2014.

[15] P. Oikonomou, A. Botsialas, A. Olziersky et al., "Wireless sensor network based on a chemocapacitive sensor array for the real-time monitoring of industrial pollutants," Procedia Engineering, vol. 87, pp. 564-567, 2014.

[16] J. R. Gisbert, C. Palau, M. Uriarte et al., "Integrated system for control and monitoring industrial wireless networks for labor risk prevention," Journal of Network and Computer Applications, vol. 39, pp. 233-252, 2014.

[17] A. Colombo, D. Fontanelli, D. Macii, and L. Palopoli, "Flexible indoor localization and tracking based on a wearable platform and sensor data fusion," IEEE Transactions on Instrumentation and Measurement, vol. 63, no. 4, pp. 864-876, 2014.

[18] Y. Ai and M. Cheffena, "On multi-hop decode-and-forward cooperative relaying for industrial wireless sensor networks," Sensors, vol. 17, no. 4, p. 695, 2017.

[19] K. Derr and M. Manic, "Wireless sensor networks-node localization for various industry problems," IEEE Transactions on Industrial Informatics, vol. 11, no. 3, pp. 752-762, 2015.

[20] M. S. Taboun, M. Gholami, and R. W. Brennan, "A distributed agent-based approach to manage industrial wireless sensor networks," IFAC-PapersOnLine, vol. 48, no. 3, pp. 1439-1444, 2015.

[21] A. Khare, R. Gupta, and P. K. Shukla, "Improving the protection of wireless sensor network using a black hole optimization algorithm (BHOA) on best feasible node capture attack," in IoT and Analytics for Sensor Networks, P. Nayak, S. Pal, and S. L. Peng, Eds., vol. 244, pp. 333-343, Springer, Singapore, 2022.

[22] X. Huang and Y. Fang, "Multiconstrained QoS multipath routing in wireless sensor networks," Wireless Networks, vol. 14, no. 4, pp. 465-478, 2007.

[23] M. Kaur, S. Singh, M. Kaur, and D. Singh, "A systematic review of metaheuristic-based image encryption techniques," Archives of Computational Methods in Engineering, 2021.

[24] Y. Gao, F. Xiao, J. Liu, and R. Wang, "Distributed soft fault detection for interval type-2 fuzzy-model-based stochastic systems with wireless sensor networks," IEEE Transactions on Industrial Informatics, vol. 15, p. 1, 2018.

[25] T. Singh, N. Saxena, M. Khurana, D. Singh, M. Abdalla, and H. Alshazly, "Data clustering using moth-flame optimization algorithm," Sensors, vol. 21, no. 12, p. 4086, 2021.

[26] Z. Sheng, C. Mahapatra, C. Zhu, and V. C. M. Leung, "Recent advances in industrial wireless sensor networks toward efficient management in IoT," IEEE Access, vol. 3, pp. 622-637, 2015.

[27] J. M. Williams, R. Khanna, J. P. Ruiz-Rosero et al., "Weaving the wireless web: toward a low-power, dense wireless sensor network for the industrial IoT," IEEE Microwave Magazine, vol. 18, no. 7, pp. 40-63, 2017.

[28] W. Sun, X. Yuan, J. Wang, Q. Li, L. Chen, and D. Mu, "Endto-End data delivery reliability model for estimating and optimizing the link quality of industrial WSNs," IEEE Transactions on Automation Science and Engineering, vol. 15, no. 3, pp. 1127-1137, 2018.

[29] T. D. Diwan, S. Choubey, H. S. Hota et al., "Feature entropy estimation (FEE) for malicious IoT traffic and detection using machine learning," Mobile Information Systems, vol. 2021, Article ID 8091363, 13 pages, 2021.
[30] X. Yang and S. Deb, "Cuckoo search via levy flights," in Proceedings of the World Congress on Nature \& Biologically Inspired Computing, Nabic, pp. 210-214, IEEE, Coimbatore, India, December 2009.

[31] S. Montero, J. Gozalvez, and M. Sepulcre, "Neighbor discovery for industrial wireless sensor networks with mobile nodes," Computer Communications, vol. 111, pp. 41-55, 2017.

[32] G. Han, A. Qian, L. Liu, J. Jiang, and C. Zhu, "Impacts of traveling paths on energy provisioning for industrial wireless rechargeable sensor networks," Microprocessors and Microsystems, vol. 39, no. 8, pp. 1271-1278, 2015.

[33] R. Kannan, M. S. Solai, and K. M. Senthil, "Pre-channel scheduling and Priority-based reservation in medium access control for industrial wireless sensor network applications," Computers \& Electrical Engineering, vol. 64, pp. 322-336, 2017.

[34] N. K. Rathore, N. K. Jain, P. K. Shukla, U. Rawat, and R. Dubey, "Image forgery detection using singular value decomposition with some attacks," National Academy Science Letters, vol. 44, no. 4, pp. 331-338, 2021.

[35] R. D. Gomes, D. V. Queiroz, A. C. Lima Filho, I. E. Fonseca, and M. S. Alencar, "Real-time link quality estimation for industrial wireless sensor networks using dedicated nodes," Ad Hoc Networks, vol. 59, pp. 116-133, 2017.

[36] D. Li, X. Li, and J. Wan, "A cloud-assisted handover optimization strategy for mobile nodes in industrial wireless networks," Computer Networks, vol. 128, pp. 133-141, 2017.

[37] D. Wang, W. Li, and P. Wang, "Measuring two-factor Authentication schemes for real-time data access in industrial wireless sensor networks," IEEE Transactions on Industrial Informatics, vol. 14, no. 9, pp. 4081-4092, 2018.

[38] S. Lu, P. Zhou, X. Wang, Y. Liu, F. Liu, and J. Zhao, "Condition monitoring and fault diagnosis of motor bearings using undersampled vibration signals from a wireless sensor network," Journal of Sound and Vibration, vol. 414, pp. 81-96, 2018.

[39] M. Kaur, V. Kumar, and D. Singh, M. Hassaballah, An efficient image steganography method using multiobjective differential evolution," Digital Media Steganography, Academic Press, Cambridge, MA, USA, pp. 65-79, 2020.

[40] A. Shrivastava, A. Rizwan, N. S. Kumar et al., "VLSI implementation of green computing control unit on zynq FPGA for green communication," Wireless Communications and Mobile Computing, vol. 2021, pp. 1-10, Article ID 4655400, 2021.

[41] S. Joshi, S. Stalin, P. K. Shukla et al., "Unified authentication and access control for future mobile communication-based lightweight IoT systems using blockchain," Wireless Communications and Mobile Computing, vol. 2021, pp. 1-12, Article ID 8621230, 2021.

[42] H. Z. Almarzouki, H. Alsulami, A. Rizwan, M. S. Basingab, H. Bukhari, and M. Shabaz, "An internet of medical thingsbased model for real-time monitoring and averting stroke sensors," Journal of Healthcare Engineering, vol. 2021, pp. 1-9, Article ID 1233166, 2021.

[43] R. Prema and R. Rangarajan, "Power aware routing protocol (PARP) for wireless sensor networks," Wireless Sensor Network, vol. 4, no. 5, pp. 133-137, 2012. 